\title{
ON THE FUZZY NATURE OF CONSTRUCTED ALGEBRAIC STRUCTURE
}

\author{
${ }^{1}$ GARBA, A. I., ${ }^{2}$ ZAKARI, Y. AND ${ }^{1}$ HASSAN, A. \\ ${ }^{1}$ Department of Mathematics, Usmanu Danfodiyo University, Sokoto. \\ ${ }^{2}$ Department of Statistics, Ahmadu Bello University, Zaria

\begin{abstract}
In this paper, some fuzzy nature of a newly constructed an algebraic structure $G_{p}(p \geq$ 5 and $p$ is always prime) were been investigated by construction of a modified fuzzy membership function on $G_{p}$ and it was used to investigate the $\propto-$ cut levelof $G_{p}$ and it was established that the $\propto$ - cut level of $G_{p}$ is the domain $G_{p} \mid w_{p-1}$, the $\operatorname{support}(\operatorname{Supp})$ of $G_{p}$ was also been investigated and we arrived at a conclusion that, the support of the $G_{p}$ structure is the entire domain of the structure $\left(G_{p}\right)$.

Keywords: fuzzy set, $\propto$ - cut level, support of a fuzzy set, permutation pattern, cycles, successors and membership function.
\end{abstract}

\subsection{INTRODUCTION}

The concept of fuzzy sets was introduced by Zadeh(1965), by defining them in terms of mappings from a set into a unit interval on the real line. Fuzzy sets were introduced to provide means to describe situations mathematically which gives rise to ill-defined classes, i.e. collection of objects for which there is no precise criteria for membership, collections of this type have a vague boundaries (Fuzzy), there are objects for which it is impossible to determine whether or not they belong to the collection. The classical mathematical theories, by which certain types of certainty can be expressed, are the classical set theory and probability theory, in terms of set theory, uncertainty is expressed by any given set of possible alternatives in situations where only one of the alternatives may actually happen. Uncertainty expressed in terms of sets of alternatives results from the non-specificity inherent in each set. Probability theory expresses uncertainty in terms of a classical measure of subsets of a given set of alternatives. The set theory introduced by Zadeh, presents the notion of membership in a given subset as a matter of degree rather than of totally in or totally out. With a fuzzy set theory, one obtains a logic in which statements may be true or false to different degrees rather than the bivalent situations (on or off) of being true or false.

Permutation pattern have been used in the past decades to study mathematical structures Audu(1986), Ibrahim(2005)studied the concept of permutation pattern using some elaborate scheme to determine the order of precedence and the position of each of the elements in a finite set of prime size have also been established in Ibrahim (2007), Garba (2018) and also an idea of embedment as an algebraic structure has yielded some interesting results by Ibrahim (2005), Garba (2018), they studied the structure and developed a scheme for the range of such cycles and use it to investigate further number theoretic and algebraic properties of $\mathbf{G}_{\mathbf{p}}$, and furthermore a group theoretic properties was also investigated by Garba (2018) and the concept of Fuzzy nature of $\mathbf{G}_{\mathbf{p}}$ has also been studied by Aremu (2017) and investigated the alpha-level cut of $\mathbf{G}_{\mathbf{p}}$. Ibrahim (2007)studied the concept of permutation pattern using some elaborate scheme to determine the order of precedence and the position for each of the elements in a finite set of prime size, and establish a scheme the scheme for generating each element in the permutation. Garba (2009) studied the $G_{p}$ structure using number theoretic properties of Catalan numbers, and also developed a scheme for range of such cycles defined to be $\left|\Delta_{f}^{l}\right|$ where $I$ is the last element in the cycle and $f$ is the first element in the cycle, and established that for all cycles in $\boldsymbol{G}_{\boldsymbol{p}}$ the range exist, they also use it to investigate further number theoretic properties of $\boldsymbol{G}_{p}$.Usman (2011)investigated the group theoretic properties of $\boldsymbol{G}_{\boldsymbol{p}}$ using composition of functions, by investigating the properties of a group and established that the structure is an Abelian group, using additive group of integers modulo $n$, where $n$ is necessarily a prime. 
BAJOPAS Volume 12 Number 1, June, 2019

Garba (2015) extended the $\boldsymbol{G}_{\boldsymbol{p}}$ structure to the $\boldsymbol{G}_{\boldsymbol{P}}{ }^{\prime}$ where a special cycle $\left(w_{p}\right)$ is introduced and the special cycle is been used as an identity of permutation and established the closure, commutativity and associativity properties from the structure. Aremu (2017)studied the $\boldsymbol{G}_{\boldsymbol{P}}{ }^{\prime}$ 'structure and investigated the $\propto-$ cut level ( $\propto$ is a fixed numerical value $\propto \in R^{+}$) of $\boldsymbol{G}_{\boldsymbol{P}}$ 'and defined the level to be $\frac{1}{p}\left(\alpha=\frac{1}{p}, \mathrm{p}\right.$ is a prime $\mathrm{p} \geq 5$ ) and established that the $\propto-$ cut level of $\boldsymbol{G}_{\boldsymbol{P}}$ 'based on $\propto=\frac{1}{p}$ is the entire domain of $\boldsymbol{G}_{\boldsymbol{p}}$.

\subsection{Preliminaries}

\subsection{Fuzzy Set}

If $X$ is a collection of object then the fuzzy set $\tilde{A} \subset X$ is a set of ordered pairs, $\tilde{A}=\left\{\left(x, \mu_{\tilde{A}}(x)\right): x \in X\right\}$, where $\mu_{\tilde{A}}(x): X \rightarrow[0,1]$ is called degree of membership of $\mathrm{x}$ in $A$ (Zadeh 1965).

\subsection{Crisp Set}

The crisp set is a set defined using characteristics functions that assign to each element of the universe a Boolean state of obedience.

\subsection{The $\propto$-Cut Level Set}

The $\propto$-level of a fuzzy set $\widetilde{A}$ is a crisp set Athatcontains the elements that have membership functions in $\widetilde{A}$ greater than or equal to $\propto$. and its represented as

$$
A_{\propto}=\left\{\propto \mid \mu_{\tilde{A}}(x) \geq \propto\right\}
$$

\subsection{The Support of a Fuzzy Set}

The support of a fuzzy set (denoted Supp) is the crisp set of all $x \in X$ for which $\mu_{\tilde{A}}(x)>0$ (Zadeh 1965).

\subsection{Cycle and Successor}

Let $\Omega$ be a non-empty, totally ordered and finite subset of $\mathbb{N}$. Let $\boldsymbol{G}_{\boldsymbol{p}}=\left\{w_{1}, w_{2}, \ldots, w_{p-1}\right\}$ be a structure such that each $w_{i}$ is generated from the arbitrary set $\Omega$ for any prime $p \geq 5$, using the scheme

$$
\begin{gathered}
w_{i}=\left((1)(1+i)_{m p}(1+2 i)_{m p} \ldots(1\right. \\
\left.+(p-1) i)_{m p}\right)
\end{gathered}
$$

Wheremp is modulop

Then each $w_{i}$ is called a cycle and the elements in each $w_{i}$ are distinct and called successors(Ibrahim 2004).

2.5.1 $n^{\text {th }}$ successor

The $n^{\text {th }}$ successor of a cycle $w_{i}$ is given by $a_{n}=(1+(n-1) i)_{m p}$

where $1 \leq n \leq p$, and $1 \leq i \leq p-1$. the number of distinct successors in a cycle is called the length of the cycle(Ibrahim 2004).

\subsubsection{Range of Cycle}

The range of a cycle $w_{i} \in \boldsymbol{G}_{\boldsymbol{p}}$ is defined as $\pi(w):=$ $\left|\Delta_{f}^{l}(w)\right|$, where $\Delta_{f}^{l}(w)$ is the difference between the first and last successor in a cycle $w$ (Garba, 2009).

2.5.3 Definition of $\boldsymbol{G}_{\boldsymbol{p}}^{\prime}$

Let $\boldsymbol{G}_{\boldsymbol{p}}=\left\{w_{1}, w_{2}, \ldots w_{p-1}\right\}$ be as defined as above then $\boldsymbol{G}_{\boldsymbol{p}}^{\prime}:=\boldsymbol{G}_{\boldsymbol{p}} \cup\left\{w_{p}\right\}$, where $w_{p}:=\{p p \ldots p\}$. that is $\boldsymbol{G}_{\boldsymbol{p}}^{\prime}=\left\{w_{1}, w_{2}, \ldots w_{p-1}, w_{p}\right\}$.

Using the above setting, if $p=5$, then we have the following set of permutations $w_{1}=(12345), w_{2}=$ (13524), $w_{3}=(14253), w_{4}=(15432)$ and this shows

that $G_{5}=\{(12345),(13524),(14253),(15432)\}$

Note that 0 and 5 are equivalent in modulo 5, thus instead of using 0 in modulo $\mathrm{p}$ we will be using p(Garba, 2009).

\subsection{RESULT AND DISCUSSION}

In this section, the discussion of the result is carried out by figures, tables and proofs.

\subsection{Fuzzy Nature of $\boldsymbol{G}_{\boldsymbol{p}}$}

Let $\boldsymbol{G}_{\boldsymbol{p}}^{\prime}:=\boldsymbol{G}_{\boldsymbol{p}} \cup\left\{w_{p}\right\}$ and $\boldsymbol{G}_{\boldsymbol{p}} \subseteq \boldsymbol{G}_{\boldsymbol{p}}^{\prime}$, then $\boldsymbol{G}_{\boldsymbol{p}}$ is a fuzzy set defined by

$$
\begin{array}{cc}
\text { Where } \mu_{\hat{G}_{p}}\left(w_{i}\right)=\left(i, \frac{\pi\left(w_{i}\right)}{p+2}\right), i<p & \hat{G}_{p}=\left\{\left(\mu_{\hat{G}_{p}}\left(w_{i}\right): w_{i} \in \boldsymbol{G}_{p}^{\prime}\right\}\right. \\
\pi\left(w_{i}\right)=\left|\Delta_{f}^{l}\left(w_{i}\right)\right|
\end{array}
$$

$\pi\left(w_{i}\right)=\left|\Delta_{f}^{l}\left(w_{i}\right)\right|$ where $l$ is the last successor and $f$ is the first successor

Illustration: consider $\boldsymbol{G}_{\boldsymbol{p}}^{\prime}$ where $\mathrm{p}=5, \boldsymbol{G}_{\mathbf{5}}^{\prime}=\left\{w_{1}, w_{2}, w_{3}, w_{4}, w_{5}\right\}$, let $\boldsymbol{G}_{\mathbf{5}} \subseteq \boldsymbol{G}_{\mathbf{5}}^{\prime}$

Then $\boldsymbol{G}_{\mathbf{5}}=\left\{w_{1}, w_{2}, w_{3}, w_{4}\right\}$, Defined $\mu_{\hat{G}_{5}}\left(w_{i}\right)=\left(i, \frac{\pi\left(w_{i}\right)}{7}\right), i<5$

$$
\begin{gathered}
\pi\left(w_{i}\right)=\left|\Delta_{f}^{l}\left(w_{i}\right)\right| \\
\mu_{\hat{G}_{5}}\left(w_{1}\right)=(1,0.6) \\
\mu_{\widehat{G}_{5}}\left(w_{2}\right)=(2,0.4) \\
\mu_{\widehat{G}_{5}}\left(w_{3}\right)=(3,0.3) \\
\mu_{\widehat{G}_{5}}(4)=(4,0.1) \\
\widehat{G}_{p}=\left\{\left(\mu_{\widehat{G}_{p}}\left(w_{i}\right)\right): w_{i} \in G_{p}^{\prime}\right\}
\end{gathered}
$$

$\hat{G}_{5}=\{(1,0.6),(2,0.4),(3,0.3),(4,0.1)\}$, then $\hat{G}_{5}$ is a fuzzy set. 
BAJOPAS Volume 12 Number 1, June, 2019

3.2 Proposition: The $\propto-$ cut level of any $G_{p}$ is $G_{p} \mid w_{p-1}$

Proof

An $\propto-$ cut level is a set that contains values from the membership functions greater than or equal to $\propto . \propto$ is an arbitrary value with the range of fuzzy $[0,1]$; let $\boldsymbol{G}_{\boldsymbol{p}}$ be a fuzzy set and $\boldsymbol{G}_{\boldsymbol{p}} \subseteq \boldsymbol{G}_{\boldsymbol{p}}^{\prime}$, then the $\propto-$ cut level is a set. $G_{p_{\alpha}}=\left\{w_{i}: \mu_{G_{p}}\left(w_{i}\right) \geq \propto\right\} \quad$ for $\propto=\frac{1}{p}$, where $(\mathrm{p} \geq 5)$

Since $G_{p_{\alpha}}=\left\{\mu_{G_{p}}\left(w_{i}\right): \mu_{G_{p}}\left(w_{i}\right) \geq \propto, i<p\right\}$

$$
\mu_{\hat{G}_{p}}\left(w_{i}\right)=\left(i, \frac{\pi\left(w_{i}\right)}{p+2}\right), i<p
$$

Without loss of generality, $\quad \mu_{\widehat{G}_{p}}\left(w_{p-1}\right)=\left(i, \frac{\pi\left(w_{p-1}\right)}{p+2}\right)$,

$$
\frac{\pi\left(w_{p-1}\right)}{p+2}<\frac{1}{p^{\prime}} \text { for any } \boldsymbol{G}_{\boldsymbol{p}} \text {. }
$$

Where $\boldsymbol{G}_{\boldsymbol{p}}=\left\{w_{1}, w_{2}, \ldots w_{p-1}\right\}$

$$
\mu_{\widehat{G}_{p}}=\left\{w_{1}, w_{2}, \ldots w_{p-2}\right\} \geq \frac{1}{p} \text {, but } \mu_{\widehat{G}_{p-1}<\frac{1}{p}} \text {. }
$$

$=>G_{p} \mid w_{p-1}$ is the domain of the alpha-cut level of the set $\widehat{G}_{p}$.

Illustration consider when $p=5$,

$$
\begin{gathered}
\boldsymbol{G}_{\mathbf{5}}=\left\{w_{1}, w_{2}, w_{3}, w_{4}\right\}, \\
\mu_{\hat{G}_{5}}\left(w_{1}\right)=(1,0.6) \\
\mu_{\hat{G}_{5}}\left(w_{2}\right)=(2,0.4) \\
\mu_{\hat{G}_{5}}\left(w_{3}\right)=(3,0.3) \\
\mu_{\hat{G}_{5}}(4)=(4,0.1) \\
\text { if } \propto=\frac{1}{p} \text {, then } \propto=\frac{1}{5}=0.2, \\
=>\quad w_{p-1}<\propto .
\end{gathered}
$$

From the illustration above it implies that, the $\propto-$ cutlevel is the domain $G_{p} \mid w_{p-1}$. The table below gives a complete description of the alpha-cut-level of the constructed algebraic structure, the alpha level of each Gp exist, and is unique.

Table 3.1: Membership Functions of $w_{i}$ and $\propto-$ Cut Level

\begin{tabular}{ccc}
\hline $\mathrm{s} / \mathrm{n}$ & $w_{i}$ & $\mu_{\widehat{G}_{p}}\left(w_{i}\right)$ \\
\hline 1 & $w_{1}$ & 0.6 \\
2 & $w_{2}$ & 0.4 \\
3 & $w_{3}$ & 0.3 \\
4 & $w_{4}$ & 0.1 \\
5 & $\propto$-cut level & 0.2 \\
\hline
\end{tabular}

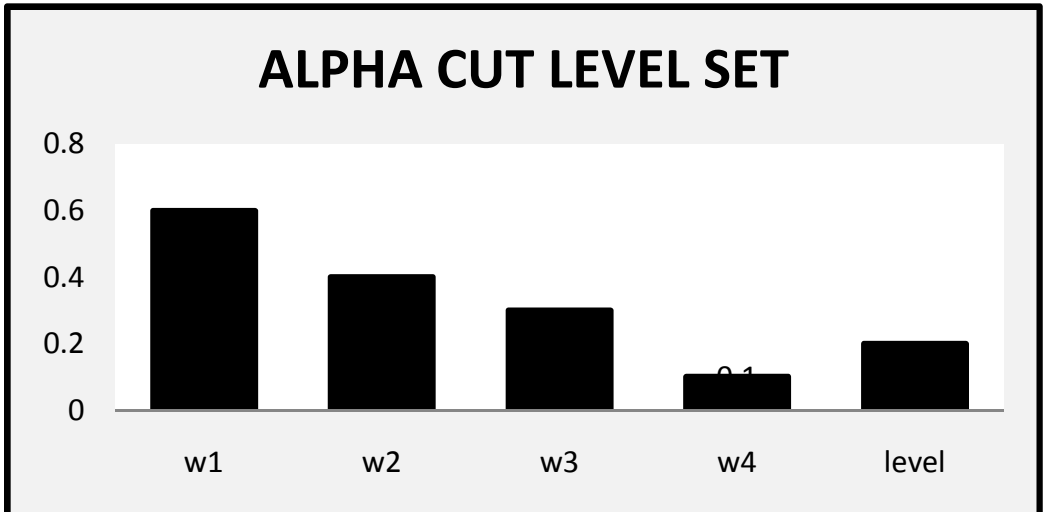

Figure 3.1: Alpha Cut Level Set

Figure 3.1 illustrate the $\propto-$ cut level of the $\mathbf{G}_{\mathbf{p}}$.the vertical axis represents the membership functions while the horizontal axis represents the permutations $w_{i}$.

For $\boldsymbol{G}_{5}$ the $\propto-$ cut level is $G_{5} \mid w_{4}$.

For $\boldsymbol{G}_{7}$ the $\propto-$ cut level is $G_{7} \mid w_{6}$.

For $\boldsymbol{G}_{\mathbf{1 1}}$ the $\propto-$ cut level is $G_{11} \mid w_{10}$.

For $\boldsymbol{G}_{13}$ the $\propto-$ cut level is $G_{13} \mid w_{12}$, e.t.c

This generalize the proof. 
3.3 Proposition: The Support of the fuzzy set $\widehat{G}_{p}$ of any $\boldsymbol{G}_{\boldsymbol{p}}$ is the entire domain.

Proof

The support of a fuzzy set (denoted by Supp) are those members of the set in which their membership degree is $>0$,

$$
\operatorname{Supp}\left(\widehat{G}_{p}\right)=\left\{\mu_{G_{p}\left(w_{i}\right)}: \mu_{G_{p}}\left(w_{i}\right)>0\right\}
$$

And $\pi\left(w_{i}\right)$ is never zero, then, the result follows.

$\operatorname{Supp}\left(\widehat{G}_{p}\right)$ is the entire domain

Table 3.2:Membership Functions of $w_{i}$

\begin{tabular}{ccc}
\hline $\mathrm{s} / \mathrm{n}$ & $w_{i}$ & $\mu_{\widehat{G}_{p}}\left(w_{i}\right)$ \\
\hline 1 & $w_{1}$ & 0.6 \\
2 & $w_{2}$ & 0.4 \\
3 & $w_{3}$ & 0.3 \\
4 & $w_{4}$ & 0.1 \\
\hline
\end{tabular}

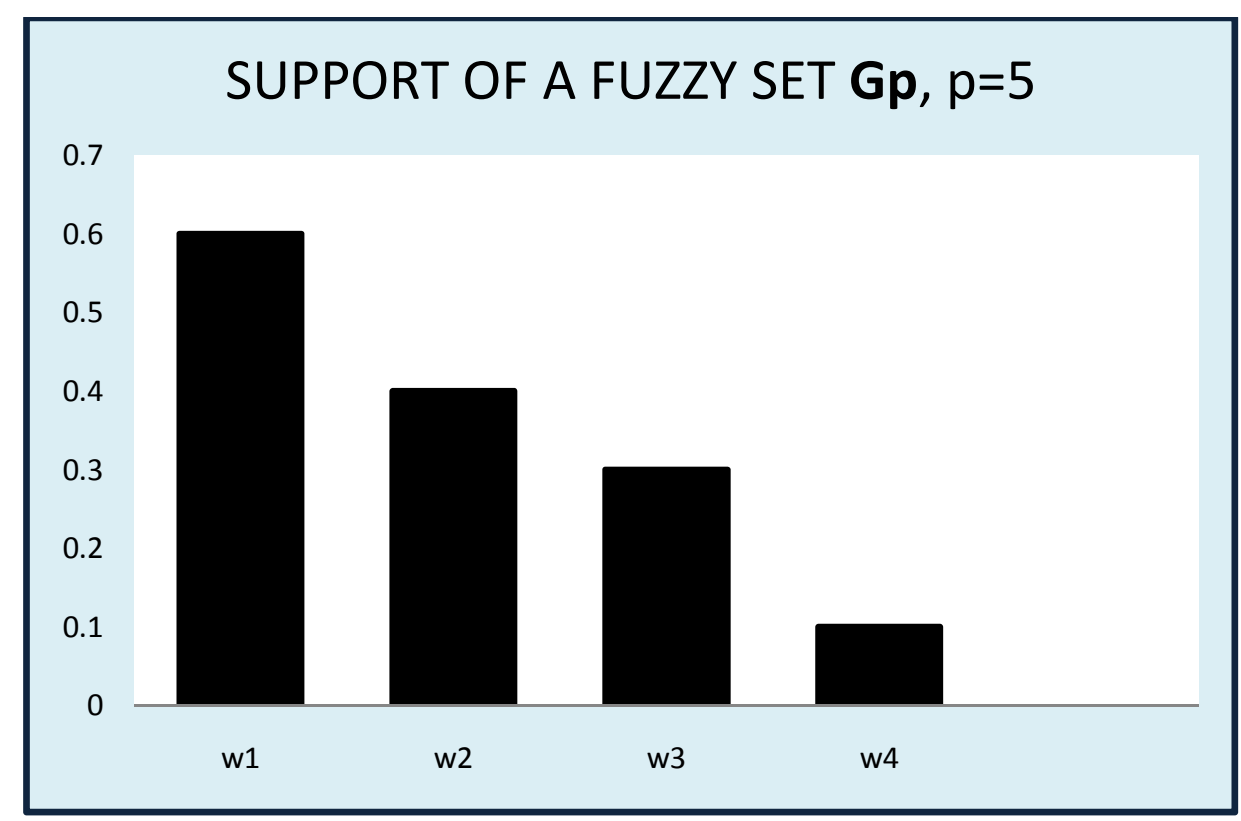

Figure 3.2: Support of a Fuzzy SetGp

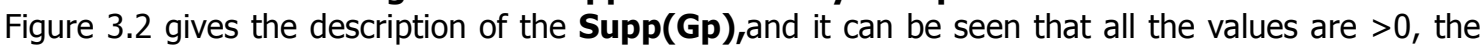
vertical axis represents the membership functions while the horizontal axis represents the permutations wand is true for any $\mathbf{G p}$, then the support of any fuzzy set in $\mathbf{G p}$ is the entire domain.

\subsection{CONCLUSION}

The construction of an algebraic structures and investigating their algebraic properties cannot be over emphasized as it has a lot of applications in different field of mathematics, in this paper we investigated some fuzzy nature of an algebraic structure $G_{p}$ that was constructed earlier, where

\section{REFERENCES}

Audu, M. S. (1986). Generating sets for Transitive Permutation Groups of Primepower Order. The Journal of Mathematical Association of Nigeria Abacus, 17(2), 22-26.

Aremu, K. O., Ejima, O. and Abdullahi, M. S.(2017). On the Fuzzy $\Gamma 1-$ non Deranged Permutation Group GГ1. Asian we discovered that if $\hat{G}_{p}$ is a fuzzy set, then the $\propto$ - cut level set of any $G_{p}$ is a set $G_{p} \mid w_{p-1}$, and the support of $\hat{G}_{p}$ is the entire domain, In the above constructed algebraic structure the first element of the permutation is always fixed.

Journal of Mathematics and Computer Research, 18, 152-157.

Cayley, A. (1889). The Collected Mathematical Papers of the Arthur Carley, II (18511860), Cambridge University Press.

Garba, A. I., Yusuf, A. and Hassan, A. (2018). Some Topological Properties of a Constructed Algebraic Structure.Journal of the Nigerian Association of Mathematical Physics, 45:21-26. 
BAJOPAS Volume 12 Number 1, June, 2019

Garba, A. I. and Ibrahim, A. A. (2009). A New Method of Constructing a Variety of Finite Group Based on Some Succession Scheme. International Journal of Physical Science, 2(3), 77-79.

Garba, A. I. and Ibrahim, A.A. (2010).A New Method of Constructing a Variety of Finite Group Based on some Succession Scheme.Internal Journal of Physical Science, 2(3), 23-26.

Garba, A. I. and Abubakar, J. (2015).Construction of an Algebraic Structure Using a Concatenation Map.Nigerian Journal of Basic and Applied Science (December, 2015), 23(2), 117-120.

Ibrahim, A. A. (2004). Group Theoretical Interpretation of Bara 'at al-Dhimmah Models for Prayers that are not Strictly Consecutive. Proceedings of Annual National Conference of the Mathematical Association of Nigeria. 35-46.

Ibrahim, A. A. and Audu, M. S. (2005). Some Group Theoretic Properties of Certain
Class of (123) and (132) Avoiding Patterns of Certain Numbers: An Enumeration Scheme. African Journal of Natural Science,8, 79-84.

Ibrahim, A. A. (2006).Correspondence between the Length of some Class of Permutation Patterns and Primitive Elements of Automophism Group Modulo n, Abacus. The Journal of Mathematical Association of Nigeria,33, 143-154.

Ibrahim, A. A. (2007). A Counting Scheme and some Algebraic Properties of a Class of Special Permutation Patterns. Journal of Discrete Mathematical Sciences and Cryptography, 10(4), 537-546.

Usman, A. and Ibrahim, A. A. (2011). A New Generating Function for Aunu Patterns: Applications in Integer Group Modulo $\mathrm{n}$. Nigerian Journal of Basic and Applied Sciences, 19(1), 1-4.

Zadeh, L. A. (1965), Calculus of Restrictions in "Fuzzy Sets and their Applications to Cognitive and Decision Processes" ( $4^{\text {th }}$., pp. 24-39). New York, Academic Press. 